Pacific Journal of Mathematics

BOUNDARY BEHAVIOR OF HOLOMORPHIC FUNCTIONS IN 


\title{
BOUNDARY BEHAVIOR OF HOLOMORPHIC FUNCTIONS IN THE BALL
}

\author{
JACOB BURBEA
}

\begin{abstract}
A description of the boundary behavior of functions belonging to certain Sobolev classes of holomorphic functions on the unit ball $B_{n}$ of $C^{n}$ is given in terms of bounded and vanishing mean oscillation. In particular, it is shown that the boundary values of any holomorphic function on $B_{n}$, whose fractional derivative of order $n / p$ belongs to the Hardy class $H^{p}\left(B_{n}\right)$, have vanishing mean oscillation provided $0<p \leq 2$.
\end{abstract}

Introduction. Let $B=B_{n}$ be the unit ball in $\mathbf{C}^{n}$ and let $H(B)$ denote the space of all holomorphic functions on $B$. By $R$ we denote the radial derivative operator $R=\sum z_{j} \partial_{j}$ where for $z=\left(z_{1}, \ldots, z_{n}\right) \in \mathbf{C}^{n}, \partial_{j}=$ $\partial / \partial z_{j}(j=1, \ldots, n)$, and we let $D_{l}=l+R$, with $D=D_{1}$, for any $l \in \mathbf{C}$. For any monomial $z^{\alpha}=z_{1}^{\alpha_{1}} \cdots z_{n}^{\alpha_{n}}, \alpha=\left(\alpha_{1}, \ldots, \alpha_{n}\right) \in \mathbf{Z}_{+}^{n}$, we have $D_{l}^{s} z^{\alpha}=(|\alpha|+l)^{s} z^{\alpha}$ for any $s \in \mathbf{Z}_{+}$, where $|\alpha|=\alpha_{1}+\cdots+\alpha_{n}$, which shows that for any $l>0, s \in \mathbf{R}$ and $f \in H(B)$, the fractional derivative $D_{l}^{s} f$ of $f$, of order $s$, is well-defined and is in $H(B)$. Let $H^{p}=H^{p}(B)$, $0<p \leq \infty$, denote the usual Hardy class of functions in $H(B)$. The Hardy-Sobolev class $H_{s}^{p}=H_{s}^{p}(B)(0<p \leq \infty, s \in \mathbf{R})$ is defined as the space of all $f$ in $H(B)$ whose fractional derivative $D^{s} f$ is in $H^{p}$, and thus $H_{0}^{p}=H^{p}$.

In the one-dimensional case $(n=1)$, most of the main properties of these $H_{s}^{p}$ spaces were investigated early by Privalov in 1918, by Hardy and Littlewood in 1932, and by Smirnov in 1932 (see the references of [7]). The question of extending these results to the higher dimensional case $(n \geq 2)$ has been considered previously by Graham [8, 9] and Krantz [10], and, quite recently, by Beatrous and Burbea [3], where these spaces are viewed as a special case of a larger family of Sobolev spaces of holomorphic functions on $B$. The following result, among other things, appears in [3]:

THEOREM 1.1. Let $0<p \leq \infty$ and $s \geq 0$.

(i) If $s>n / p$ then $H_{s}^{p}$ is contained in the Lipschitz class $\Lambda_{s-n / p}(B)$;

(ii) If $0 \leq s<n / p$ then $H_{s}^{p}$ is contained in the Hardy class $H^{p n /(n-p s)}(B)$; 
(iii) If $s=n / p$ then $H_{s}^{p}$ is contained in $\operatorname{BMOA}(B)$, the class of functions in $H(B)$ with bounded mean oscillation.

In the one-dimensional case $(n=1)$, this theorem is classical for $s=1$; assertions (i) and (ii) are due to Hardy and Littlewood (see [7, pp. 88-91] and assertion (iii) is due to Privalov (see [7, pp. 42-52]). Moreover, assertion (iii) in this particular case, of $s=1$ and the unit disk $\Delta=B_{1}$, admits even a stronger form. In fact, by Privalov's results $H_{1}^{1}(\Delta)=\mathrm{AC}(\Delta)$, where $\mathrm{AC}(\Delta)$ is the class of all functions in $H(\Delta)$ which are continuous on $\bar{\Delta}$ and absolutely continuous on $\partial \Delta$. In the higher-dimensional case $(n \geq 2)$ and $s=1$, assertions (i) and (ii) of the above theorem are due to Graham [9] and Krantz [10], while assertion (iii) has been conjectured in Graham [8] and was later proved by Krantz [10].

The main purpose of the present paper is to refine assertion (iii) by obtaining better boundary behavior when $p \leq 2$. This refinement will be modelled after the following one-dimensional result whose proof appears in [3]:

TheOREM 1.2. Let $0<p \leq 2$ and $s=1 / p$. Then $H_{s}^{p}(\Delta)$ is contained in $\operatorname{VMOA}(\Delta)$, the class of functions in $H(\Delta)$ with vanishing mean oscillation. If also $0<p \leq 1$ then $H_{s}^{p}(\Delta)$ is contained in the Privalov class $\operatorname{AC}(\Delta)$.

As mentioned previously, for $p=1$ this theorem is Privalov's result $H_{1}^{1}(\Delta)=\mathrm{AC}(\Delta)$. To find the precise analogue of the Privalov class when $n \geq 2$, so that it equals the class $H_{n}^{1}(B)$, seems to be rather difficult. A reasonable analogue is the class $\mathrm{AC}(B)$ consisting of all functions in the ball-algebra $A(B)$ which are absolutely continuous on any real analytic curve in $\partial B$ which is nowhere complex tangential. Here $A(B)$ is the Banach algebra of functions in $H(B)$ which are continuous on $\bar{B}$ and normed by the sup-norm. Under these circumstances, Beatrous, in a very recent paper [2], was able to show that $H_{n}^{1}(B)$ is contained in $\mathrm{AC}(B)$. The proof in [2] is based on Privalov's result and on results from [3] as well as [1]; for sake of completeness a slightly different proof is provided also here. For $p=2$, the space $H_{1 / 2}^{2}(\Delta)$ appearing in Theorem 1.2 is equivalent to the space $\mathscr{D}(\Delta)$ of holomorphic functions with a finite Dirichlet integral on $\Delta$ which, by a result of Stegenga [14], forms a subspace of $\operatorname{VMOA}(\Delta)$. Thus, in effect, for $p=2$ this theorem is a reformulation of Stegenga's result. In this paper, we extend Stegenga's result to higher dimensions $n \geq 2$ by showing that $H_{n / 2}^{2}(B)$ is contained in $\operatorname{VMOA}(B)$, 
the class of functions in $H(B)$ with vanishing mean oscillation. In fact, we shall identify $H_{n / 2}^{2}(B)$ with the so-called Hilbert-Schmidt space $\mathrm{HS}(B)$ on $B$, which is a proper subspace of $\operatorname{VMOA}(B)$ consisting of all $g \in H(B)$ for which the associated Hankel operator $K_{g}$ is a Hilbert-Schmidt operator on $H^{2}(B)$ (see Theorem 4.1). We may now formulate the refinement of assertion (iii) of Theorem 1.1 in the following form:

THEOREM 1.3. Let $0<p \leq \infty$ and $s=n / p$.

(i) If $0<p \leq \infty$ then $H_{s}^{p}(B)$ is contained in $\mathrm{BMOA}(B)$;

(ii) If $0<p \leq 2$ then $H_{s}^{p}(B)$ is contained in $\mathrm{HS}(B)$;

(iii) If $0<p \leq 1$ then $H_{s}^{p}(B)$ is contained in $\mathrm{AC}(B)$.

In this theorem, assertion (i) is a special case of a result in [3], assertion (ii) is a special case of Theorem 2.8 (ii) of this paper, and assertion (iii) is a special case of a recent result of Beatrous [2] (see Theorem 2.8 (iii) of this paper). Graham [8] has constructed an example in the unit ball $B_{2}$ of $C^{2}$ of an unbounded function in $H_{1}^{2}\left(B_{2}\right)$, thus showing that in general one cannot expect much improvement in assertion (i) of Theorem 1.3. This result has been refined by Beatrous [2] in showing that for $p>1$ and $s=n / p$, the space $H_{s}^{p}(B)$ contains unbounded functions. In this paper we give a further refinement (see Theorem 5.3 below) of Graham and Beatrous results by also showing that when $s=n / p$ the space $H_{s}^{p}(B)$ contains unbounded functions which belong to $\operatorname{HS}(B)$ if $p>1$ and it contains unbounded functions which are not in $\operatorname{HS}(B)$ if $p>2$.

Throughout the paper, $c$ denotes an absolute positive constant whose value may change from one occurrence to the other but is independent of the relevant parameters in the expression in which it occurs. Moreover, for two complex-valued functions $f$ and $g$ on a non-void set $\Lambda$, we use the notation $f \simeq g$ on $\Lambda$ to mean $c^{-1}|g(\lambda)| \leq|f(\lambda)| \leq c|g(\lambda)|$ for every $\lambda \in \Lambda$.

2. Preliminaries and background. For $z=\left(z_{1}, \ldots, z_{n}\right) \in \mathbf{C}^{n}$, we let $u_{j}(z)=z_{j}(1 \leq j \leq n)$, and hence $u_{j}$ is the orthogonal projection of $\mathbf{C}^{n}$ onto the $j$ th complex coordinate axis. The inner product on $\mathbf{C}^{n}$ is denoted by $\langle$,$\rangle , where$

$$
\langle z, \zeta\rangle=z_{1} \bar{\xi}_{1}+\cdots+z_{n} \bar{\zeta}_{n} .
$$

The norm is then $\|z\|=\sqrt{\langle z, z\rangle}$, and thus $B=\left\{z^{\prime} \in \mathbf{C}^{n}:\|z\|<1\right\}$. For $\alpha=\left(\alpha_{1}, \ldots, \alpha_{n}\right) \in \mathbf{Z}_{+}^{n}$ we use the usual multi-index conventions of $\alpha ! \cdots$ $\alpha_{n}$ !, and $\partial^{\alpha}=\partial_{1}^{\alpha_{1}} \cdots \partial_{n}^{\alpha_{n}}$. The Lipschitz class $\Lambda_{s}=\Lambda_{s}(B)$, of order 
$s>0$, is defined as follows: For $0<s \leq 1, \Lambda_{s}$ is the class of functions $f$ in $H(B)$ satisfyisng $|f(z+\zeta)+f(z-\zeta)-2 f(z)| \leq c\|\zeta\|^{s}$ whenever $z$ and $z \pm \zeta$ are in $B$. For non-integer $s>1$, we define $\Lambda_{s}$ to be the class of functions $f$ in $H(B)$ with $\partial^{\alpha} f \in \Lambda_{s-[s]}$ whenever $|\alpha| \leq[s]$, where $[s]$ denotes the integer-value of $s$. Finally, if $s$ is a positive integer then $\Lambda_{s}$ is defined as the class of functions $f$ in $H(S)$ with $\partial^{\alpha} f \in \Lambda_{1}$ whenever $|\alpha| \leq s-1$.

Following $[3,4]$ we consider a family $\left\{d v_{q}\right\}, q>0$, of probability measures on $\bar{B}$, defined by

$$
d v_{q}(z)=\pi^{-n} \frac{\Gamma(n+q)}{\Gamma(q)}\left(1-\|z\|^{2}\right)^{q-1} d v(z)
$$

where $d v$ is the usual Lebesgue measure on $\mathbf{C}^{n}$. It follows by integration in polar coordinates that as $q \rightarrow 0^{+}$, the measures $d v_{q}$ on $\bar{B}$ converge weakly to the normalized surface measure $d \sigma$ on $\partial B$, and thus we may define $d v_{0}$ as $d \sigma$. For $0<p \leq \infty$ we denote by $L_{q}^{p}$ the $L^{p}$-space with respect to the measure $d v_{q}, q \geq 0$, and we let $\|\cdot\|_{p, q}$ denote the associated norm. Thus

$$
\|f\|_{p, q}=\left\{\int|f|^{p} d v_{q}\right\}^{1 / p} \quad(0<p<\infty)
$$

with the usual sup-norm convention when $p=\infty$. Note that for $0<p<1$ the term "norm" used here for $\|\cdot\|_{p, q}$ is abused, however in this case $\rho(f, g)=\|f-g\|_{p, q}^{p}$ defines a metric on $L_{q}^{p}$ which turns it into a complete topological vector space. We let $A_{q}^{p}=A_{q}^{p}(B)$ denote the subspace of $L_{q}^{p}$ consisting of holomorphic functions on $B$. In particular, when $q=0$, we obtain the Hardy class $H^{p}=A_{0}^{p}$, which we identify in the usual way as a subspace of $L_{0}^{p}=L_{0}^{p}(\partial B)$. Moreover, for any $f$ in $H(B)$ we have

$$
\|f\|_{p, 0}=\sup _{0<r<1}\left\{\int|f(r z)|^{p} d v_{0}(z)\right\}^{1 / p}
$$

and the norms $\|f\|_{p, q}$ converge to $\|f\|_{p, 0}$ as $q \rightarrow 0^{+}$. From this follows that $H^{p}$ may be viewed as an inductive limit of the spaces $A_{q}^{p}$ as $q \rightarrow 0^{+}$.

By $\langle,\rangle_{q}$ we denote the inner product on $L_{q}^{2}$, thus

$$
\langle f, g\rangle_{q}=\int f \bar{g} d v_{q} \quad(q \geq 0) .
$$

It follows that $A_{q}^{2}$ is a functional Hilbert space of holomorphic functions on $B$, with the reproducing kernel

$$
k_{q}(z, \zeta)=(1-\langle z, \zeta\rangle)^{-(n+q)} \quad(z, \zeta \in B),
$$

and with $\left\{\sqrt{\Gamma(n+q+|\alpha|) / \alpha ! \Gamma(n+q)} z^{\alpha}: \alpha \in \mathbf{Z}_{+}^{n}\right\}$ as an orthonormal basis (see $[4,5])$. The orthogonal projection on $L_{q}^{2}$ onto $A_{q}^{2}$ is denoted by 
$P_{q}$, and thus

$$
\left\{P_{q} f\right\}(z)=\left\langle f, k_{q}(\cdot, z)\right\rangle_{q}=\int f(\zeta) k_{q}(z, \zeta) d v_{q}(\zeta) .
$$

With the projector $P_{q}$ we associate the Hankel operator $K_{g}^{(q)}$, with the symbol $g \in A_{q}^{2}$, defined on $A_{q}^{2}$ by $K_{q}^{(q)}(f)=P_{q}(g \bar{f})$. Thus $K_{g}^{(q)}$ is a conjugate-linear operator in $A_{q}^{2}$. When $q=0$ we write simply $P$ and $K_{g}$ for $P_{0}$ and $K_{g}^{(0)}$, respectively.

We shall need some properties of the spaces $\mathrm{BMOA}=\mathrm{BMOA}(B)$ and $\operatorname{VMOA}=\operatorname{VMOA}(B)$. Most of these properties may be found in Coifman, Rochberg and Weiss [6], and in Sarason [13]. The space BMOA consists of all $f \in A_{0}^{2}$ such that

$$
\|f\|_{\mathrm{BMOA}} \equiv \sup \left\{\left|\langle f, g\rangle_{0}\right|: g \in A_{0}^{2},\|g\|_{1,0}=1\right\}<\infty .
$$

Evidently, BMOA is a Banach space with the norm $\|\cdot\|_{\mathrm{BMOA}}$ and it serves as a dual to $A_{0}^{1}$, with the duality realized by the pairing $\lim _{r \rightarrow 1^{-}}\left\langle f, g_{r}\right\rangle_{0}$, where $g_{r}(z) \equiv g(r z) \quad(z \in B, 0<r<1)$. Moreover, we have $A_{0}^{\infty} \subset$ BMOA $\subset A_{0}^{p}, 0<p<\infty$, and the injections are continuous. We also have (see [6]):

THEOREM 2.1. For $g \in A_{0}^{2}$ the following conditions are equivalent:

(i) $g \in \mathrm{BMOA}$;

(ii) $g=P(f)$ for some $f \in L_{0}^{\infty}$

(iii) $K_{g}$ maps $A_{0}^{2}$ continuously into $A_{0}^{2}$.

Moreover, if any one of these conditions hold then $f$ can be chosen so that $\|f\|_{0, \infty} \simeq\|g\|_{\mathrm{BMOA}} \simeq\left\|K_{g}\right\|$.

The next result is rather well-known, its proof is included for sake of completeness. For simplicity, the norm $\|\cdot\|_{\infty, 0}$ of $L_{0}^{\infty}$ will be denoted by $\|\cdot\|_{\infty}$.

LEMMA 2.2. For $f \in H(B)$, we have $\|f\|_{\mathrm{BMOA}} \leq 2\|\operatorname{Im} f\|_{\infty}+|f(0)|$.

Proof. From the definition of the BMOA-norm and the properties of the projector $P$, we have that $\|1\|_{\mathrm{BMOA}}=1$ and that $\|P(g)\|_{\mathrm{BMOA}} \leq\|g\|_{\infty}$ for every $g \in L_{0}^{\infty}$. In particular, if $f \in H(B)$ then $2 i P(\operatorname{Im} f)=P f-P \bar{f}=$ $f-\overline{f(0)}$, and thus $\|f-\overline{f(0)}\|_{\mathrm{BMOA}} \leq 2\|\operatorname{Im} f\|_{\infty}$. The result now follows from the triangle inequality.

The space VMOA is a subspace of BMOA consisting of all $g$ in BMOA so that $g=P(f)$ for some $f \in C(\partial B)$. Alternatively, VMOA can be characterized as the BMOA-closure of the polynomials and hence also 
of $A(B)$, the class of all functions in $H(B)$ which are continuous on $\bar{B}$. We also have (see [6]):

TheOREM 2.3. Let $g \in H(B)$. Then $g \in \mathrm{VMOA}$ if and only if $K_{g}$ is a compact operator on $A_{0}^{2}$.

In light of this theorem, we define the Hilbert-Schmidt space $\operatorname{HS}(B)$ as the class of all $g \in H(B)$ so that $K_{g}$ is a Hilbert-Schmidt operator on $A_{0}^{2}$. Evidently, this is a proper subspace of VMOA and $\left\|K_{g}\right\| \leq\left\|K_{g}\right\|_{2} \leq$ $\|g\|_{\text {BMOA }}$ where $\left\|K_{g}\right\|_{2}$ denotes the Hilbert-Schmidt operator-norm of $K_{g}$, $g \in H S(B)$. We also consider the (little) Bloch space $\mathscr{B}_{0}=\mathscr{B}_{0}(B)$ consisting of all $f \in H(B)$ so that $|\{D f\}(z)|=o\left(\left(1-\|z\|^{2}\right)^{-1}\right)$ as $\|z\| \rightarrow 1^{-}$, and we let $\mathrm{BMOA}^{0}=\mathscr{B}_{0} \cap \mathrm{BMOA}$. Evidently, VMOA is also a subspace of $\mathrm{BMOA}^{0}$.

For $s \in \mathbf{Z}_{+}, q \geq 0$ and $0<p \leq \infty$, we consider the weighted Sobolev space $\mathscr{W}_{q, s}^{p}=\mathscr{W}_{q, s}^{p}(B)$ of holomorphic functions $f$ on $B$ such that $\partial^{\alpha} f \in A_{p}^{p}$ for every $\alpha \in \mathbf{Z}_{+}^{n}$ with $|\alpha| \leq s$. The norm of $f \in \mathscr{W}_{q, s}^{p}$ may be given by

$$
\|\| f \|_{p, q ; s}=\left\{\sum_{|\alpha| \leq s}\left\|\partial^{\alpha} f\right\|_{p, q}^{p}\right\}^{1 / p}
$$

Although this definition of a Sobolev norm is standard, it is more convenient for our purposes to employ equivalent norms which involve derivatives in only the radial direction. Accordingly, for $s \in \mathbf{R}, q \geq 0$, and $0<p \leq \infty$ we set

$$
\|f\|_{p, q ; s}=\left\|D^{s} f\right\|_{p, q}
$$

whenever $f \in H(B)$, and we let $A_{q, s}^{p}=A_{q, s}^{p}(B)=\left\{f \in H(B):\|f\|_{p, q ; s}<\right.$ $\infty\}$. It follows that $\mathscr{W}_{q, 0}^{p}=A_{q, 0}^{p}=A_{q}^{p}$ and that $A_{0, s}^{p}=H_{s}^{p}$, the HardySobolev class mentioned in the introduction. The proof of the following theorem appears in [3]:

TheOREM 2.4. Let $q \geq 0,0<p \leq \infty$, and $s \in \mathbf{Z}_{+}$. Then $A_{q, s}^{p}=\mathscr{W}_{q, s}^{p}$ and their norms are equivalent.

For $p=2$, the space $A_{q, s}^{p}$ is of special interest. In this case $A_{q, s}^{2}$ is a functional Hilbert space of holomorphic functions on $B$ with a reproducing kernel $k_{q, s}$ given by

$$
k_{q, s}(z, \zeta)=G_{n+q, 2 s}(\langle z, \zeta\rangle) \quad(z, \zeta \in B),
$$

where $G_{a, b}$ is a holomorphic function on the unit disk $\Delta$ with the expansion

$$
G_{a, b}(\lambda)=\sum_{m=0}^{\infty} \frac{1}{m !} \frac{(a)_{m}}{(m+1)^{b}} \lambda^{m} \quad(\lambda \in \Delta),
$$


and where

$$
(a)_{m}=\Gamma(m+a) / \Gamma(a)=a(a+1) \cdots(a+m-1) .
$$

In particular, for any $f \in A_{q, s}^{2}$ and any $z \in B$, we have

$$
f(z)=\left\langle D^{s} f, G_{n+q, s}(\langle\cdot, z\rangle)\right\rangle_{q} .
$$

Moreover, for any $f \in H(B)$ with $f(z)=\sum a_{\alpha} z^{\alpha}$ we have

$$
\|f\|_{2, q ; s}^{2}=\sum_{\alpha} \frac{\alpha !(|\alpha|+1)^{2 s}}{(n+q)_{|\alpha|}}\left|a_{\alpha}\right|^{2} .
$$

From this follows (see [3]) that when $q_{1}-q_{2}=2\left(s_{1}-s_{2}\right)$, the spaces $A_{q_{1}, s_{1}}^{2}$ and $A_{q_{2}, s_{2}}^{2}$ are equal and their norms are equivalent. This result admits an extension to the case $p \neq 2$ in the form of the following two theorems, the proofs of which can be found in [3].

Theorem 2.5. Let $0<p \leq \infty, q_{J} \geq 0$ and $s_{j} \in \mathbf{R}(j=1,2)$ such that $\left(q_{1}-q_{2}\right) / p=s_{1}-s_{2}$.

(i) If $0<p \leq \infty$ and $q_{j} \geq 0(j=1,2)$, then $A_{q_{1}, s_{1}}^{p}=A_{q_{2}, s_{2}}^{p}$ and their norms are equivalent;

(ii) If $2 \leq p \leq \infty$ and $q_{2}=0$, then $A_{0, s_{2}}^{p} \subset A_{q_{1}, s_{1}}^{p}$ and the inclusion is continuous;

(iii) If $0<p \leq 2$ and $q_{2}=0$, then $A_{q_{1}, s_{1}}^{p} \subset A_{0, s_{2}}^{p}$ and the inclusion is continuous.

THEOREM 2.6. Let $0<p_{1}<p_{2}<\infty, q_{j} \geq 0$ and $s_{j} \in \mathbf{R}(j=1,2)$ such that $\left(n+q_{1}\right) / p_{1}-\left(n+q_{2}\right) / p_{2}=s_{1}-s_{2}$. Then $A_{q_{1}, s_{1}}^{p_{1}} \subset A_{q_{2}, s_{2}}^{p_{2}}$ and the inclusion is continuous.

The next theorem is a generalization of Theorem 1.1 when $q>0$ and forms a slight refinement of assertion (iii) there. This theorem, whose proof appears in [3], describes the boundary behavior of functions in $A_{q, s}^{p}$.

TheOREM 2.7. Let $0<p \leq \infty, q \geq 0$ and $s \geq 0$. tinuous;

(i) If $s>(n+q) / p$ then $A_{q, s}^{p} \subset \Lambda_{s-(n+q) / p}$ and the inclusion is con-

(ii) If $0 \leq s<(n+q) / p$ then $A_{q, s}^{p} \subset A_{q}^{p(n+q) /[(n+q)-p s]}$ and the inclusion is continuous;

(iii) If $s=(n+q) / p$ then $A_{q, s}^{p} \subset \mathrm{BMOA}^{0}$ and the inclusion is continuous. 
Assertion (iii) of this theorem admits a further refinement when $p \leq 2$, a refinement generalizing Theorem 1.3, announced in the introduction, to include the case $q \geq 0$. This refinement may be stated as follows:

THEOREM 2.8. Let $0<p \leq \infty, q \geq 0$ and $s=(n+q) / p$.

(i) If $0<p \leq \infty$ then $A_{q, s}^{p} \subset \mathrm{BMOA}^{0}$ and the inclusion is continuous;

(ii) If $0<p \leq 2$ then $A_{q, s}^{p} \subset \mathrm{HS}(B)$ and the inclusion is continuous;

(iii) If $0<p \leq 1$ then $A_{q, s}^{p} \subset \mathrm{AC}(B)$ and the inclusion is continuous.

In this theorem, assertion (iii) was first proved in Beatrous [2] by reducing the general case to the one-dimensional case which is already known by virtue of the classical result of Privalov. For sake of completeness we provide a slightly different proof (see Theorem 3.6) which is based on a rather general result (see Theorem 3.3) and on a previous result in Beatrous [1] (see Lemma 3.5). Assertion (i) is assertion (iii) of Theorem 3.7, while assertion (ii), which may be regarded as one of the main results of this paper, is proved in $\S 4$. The fact that the refinement provided in Theorem 2.8 is sharp will be established in Theorem 5.3, special cases of which were previously considered also in Graham [8] and Beatrous [2]. Another, perhaps interesting, result of this paper is Theorem 4.1 which shows, in particular, that the Hilbert-Schmidt class $\operatorname{HS}(B)$, which is a proper subspace of VMOA, is completely equivalent to every weighted Sobolev space $A_{q,(n+q) / 2}^{2}, q \geq 0$.

3. Continuous extensions. In this section we discuss the possibility of extending certain bounded functions on $B$ to continuous functions on $\bar{B}$. To this end we shall use the following representation formula which is a special case of formula (2.2) and a result in [3] concerning the asymptotic behavior of the function $G_{a, b}$, defined in (2.1).

LEMMA 3.1. For any $q \geq 0$, the function $G_{q} \equiv G_{n+q, n+q}$ on $\Delta$ admits the following properties:

(i) $G_{q}(\lambda)=-\{\Gamma(n+q) \lambda\}^{-1} \log (1-\lambda)+F_{q}(\lambda), \lambda \in \Delta$, where $F_{q} \in$ $\Lambda_{1}(\Delta)$ with $F_{q}(0)=1-\{\Gamma(n+q)\}^{-1}$;

(ii) $\left\|\operatorname{Im} G_{q}\right\|_{\infty} \leq c(q)$ for some positive constant $c(q)$;

(iii) For every $f \in A_{q, n+q}^{1}$ and every $z \in B$,

$$
f(z)=\int\left\{D^{n+q} f\right\}(\zeta) G_{q}(\langle z, \zeta\rangle) d v_{q}(\zeta) .
$$

COROLlary 3.2. For any $q \geq 0$ and any $\zeta \in B, \| G_{q}\left(\langle\cdot, \zeta\rangle \|_{\mathrm{BMOA}} \leq\right.$ $2 c(q)+1$. 
Proof. This follows from (ii) of the above lemma and Lemma 2.2.

For $q \geq 0$, we define

$$
K_{q}(z, \zeta)=1+2 i \operatorname{Im} G_{q}(\langle z, \zeta\rangle) \quad(z, \zeta \in B)
$$

Theorem 3.3. For $q \geq 0$, let $\left\{T_{q} f\right\}(z)=\left\langle f, K_{q}(\cdot, z)\right\rangle_{q}$. Then $T_{q}$ is a continuous linear transformation of $L_{q}^{1}$ into $L_{q}^{\infty}$ and of $A_{q}^{1}$ into $A_{q}^{\infty} \equiv H^{\infty}$ with norm $\left\|T_{q}\right\| \leq 2 c(q)+1$. Moreover, $T_{q} f$ has a continuous extension to $\bar{B}$ for every $f \stackrel{\varphi}{\in} L_{q}^{1}$.

Proof. For $f \in L_{q}^{1}$, we have, by Lemma 3.1 (ii) and (3.1), that $\left\|T_{q} f\right\|_{\infty, q} \leq(2 c(q)+1)\|f\|_{1, q}$. If also $f \in A_{q}^{1}$ then by the properties of the orthogonal projection $P_{q}$ we have

$$
\left\{T_{q} f\right\}(\zeta)=\left\langle P_{q} f, K_{q}(\cdot, z)\right\rangle_{q}=\left\langle f, P_{q} K_{q}(\cdot, z)\right\rangle_{q}=\left\langle f, G_{q}(\langle\cdot, z\rangle)\right\rangle_{q},
$$

and hence $T_{q} f \in H(B)$. This proves the first part of theorem. To prove the second part, we first note that by virtue of Lemma 3.1 (ii) and (3.1), $\left\|K_{q}(\cdot, z)\right\|_{\underline{\infty}, q} \leq 2 c(q)+1$ for all $z$ in $\bar{B}$. Let $\left\{z_{m}\right\}$ be a sequence of points in $\bar{B}$ with $z_{m} \rightarrow z$. It follows from the Banach-Alaoglu theorem that the sequence $\left\{K_{q}\left(\cdot, z_{m}\right)\right\}$ has a weak* convergent subsequence in $L_{q}^{\infty}$. Without any loss of generality, we may assume that this subsequence is the sequence $\left\{K_{q}\left(\cdot, z_{m}\right)\right\}$ itself. Now, since $\left\{K_{q}\left(\cdot, z_{m}\right)\right\}$ is a uniformly bounded sequence in $L_{q}^{\infty}$ we find that $K_{q}\left(\cdot, z_{m}\right) \rightarrow K_{q}(\cdot, z)$ pointwise on $B$ and almost everywhere on $\partial B$, and thus, by the Lebesgue dominated convergence theorem, $\left\{K_{q}\left(\cdot, z_{m}\right)\right\}$ is a sequence in $L_{q}^{\infty}$ which is weak* convergent to $K_{q}(\cdot, z)$. From this follows that the mapping $z \mapsto K_{q}(\cdot, z)$ is weak* continuous as a mapping from $\bar{B}$ into $L_{q}^{\infty}$. In particular, for any $f \in L_{q}^{1}$, the mapping $z \mapsto\left\{T_{q} f\right\}(z)=\left\langle f, K_{q}(\cdot, z)\right\rangle_{q}$ is continuous on $\bar{B}$, and the proof is complete.

Corollary 3.4. Let $q \geq 0$ and $f \in A_{q, n+q}^{1}$. Then $f$ is continuous on $\bar{B}$ with $\|f\|_{\infty, q} \leq[2 c(q)+1]\|f\|_{1, q ; n+q}$.

Proof. Set $g=D^{n+q} f$, and hence $g \in A_{q}^{1}$. By Lemma 3.1 (iii) and (3.1),

$$
f(z)=\left\langle g, G_{q}(\langle\cdot, z\rangle)\right\rangle_{q}=\left\langle g, P_{q} K_{q}(\cdot, z)\right\rangle_{q}=\left\{T_{q} g\right\}(z),
$$

and the result follows from Theorem 3.3. 
Let $D$ be a bounded smooth domain in $\mathbf{C}^{n}$ and let $s \in \mathbf{Z}_{+}$. We will denote by $H_{s}^{p}(D)$ the space of all holomorphic functions on $D$ with partial derivatives up to order $s$ in the Hardy class $H^{p}(D), 0<p \leq \infty$. We shall need the following special case of a result in Beatrous [1]:

LeMma 3.5. Let $D$ be a smoothly bounded strictly pseudoconvex domain in $\mathbf{C}^{n}$, and let $M$ be a one-dimensional complex submanifold of a neighborhood of $\bar{D}$ which meets $\partial D$ transversally. Then for any $f \in H_{n}^{1}(D)$ the restriction to $M$ of the partial derivatives $\partial_{j} f, 1 \leq j \leq n$, are in $H^{1}(M \cap D)$.

We are now in a position to prove assertion (iii) of Theorem 2.8 due to Beatrous [2]. The present proof is only a slight variation to Beatrous' proof, and is included here for sake of completeness.

THEOREM 3.6. Let $0<p \leq 1, q \geq 0$ and $s=(n+q) / p$. Then $A_{q, s}^{p} \subset$ $\mathrm{AC}(B)$ and the inclusion is continuous.

Proof. We first observe that, by Theorem 2.5 (iii) and Theorem 2.6, we have $A_{q, s}^{p} \subset A_{0, n}^{1}$ and the inclusion is continuous. Next, by Corollary 3.4, any $f \in A_{0, n}^{1}$ is continuous on $\bar{B}$ with $\|f\|_{\infty} \leq[2 c(0)+1]\left\|D^{n} f\right\|_{1,0}$. Thus, it is sufficient to show that the restriction of any function in $A_{0, n}^{1}$ to any non-tangential real analytic curve in $\partial B$ is absolutely continuous. Let $f$ and $\Gamma$ be such a function and such a curve. Let $\zeta$ be an arbitrary point of $\Gamma$. Since absolute continuity is a local property, it suffices to show that the restriction of $f$ to a small neighborhood of $\zeta$ is absolutely continuous. Choose a one-dimensional complex submanifold $M$ of a neighborhood $N$ of $\zeta$ with $M \cap N=\Gamma \cap N$, and a strictly pseudoconvex domain $D$ with smooth boundary such that $\bar{D} \subset \bar{B} \cap N$, and such that $\partial D$ contains a neighborhood of $\zeta$ in $\Gamma$. By choosing $D$ sufficiently small, we may also assume that $M$ meets $\partial D$ transversally and that $M \cap D$ is simply connected. It follows that there exists a $C^{\infty}$-diffeomorphism $\phi: \bar{\Delta} \rightarrow M \cap$ $\bar{D}$ which is holomorphic on $\bar{\Delta}$, and such that $\phi(1)=\zeta$. Thus, by Lemma 3.5 and the chain rule, $(f \circ \phi)^{\prime}$ is in the Hardy class $H^{1}(\Delta)$ and consequently, by Privalov's classical result, the restriction to $\partial \Delta$ of $f \circ \phi$ is absolutely continuous. It follows, since $\phi$ is a diffeomorphism of a neighborhood of 1 in $\partial \Delta$ into a neighborhood of $\zeta$ in $\Gamma$, that $\left.f\right|_{\Gamma}$ is absolutely continuous in a neighborhood of $\zeta$ in $\Gamma$, and the proof is complete.

4. Hilbert-Schmidt space. For $q \geq 0$ and $g \in A_{q}^{2}$ we consider the previously defined Hankel operator $K_{g}^{(q)}$, and define the space $\operatorname{HS}_{q}(B)$ as the class of all $g \in A_{q}^{2}$ so that $K_{g}^{(q)}$ is a Hilbert-Schmidt operator on $A_{q}^{2}$. 
We recall that when $q=0, K_{g}^{(0)}=K_{g}$ and $\mathrm{HS}_{0}(B)$ is the Hilbert-Schmidt space $\mathrm{HS}(B)$. Moreover, $\mathrm{HS}(B) \subset \mathrm{VMOA} \subset \mathrm{BMOA}^{0}$ with $\|g\|_{\mathrm{BMOA}} \simeq$ $\left\|K_{g}\right\| \leq\left\|K_{g}\right\|_{2}$ for every $g \in H(B)$, where $\left\|K_{g}^{(q)}\right\|_{2}$ denotes the HilbertSchmidt norm of the operator $K_{g}^{(q)}$. The next result shows that, in fact, $\mathrm{HS}_{q}(B)=\mathrm{HS}(B)=A_{q,(n+q) / 2}^{2}=A_{0, n / 2}^{2}$ with equivalent norms, i.e.

$$
\left\|K_{g}^{(q)}\right\|_{2} \simeq\left\|K_{g}\right\|_{2} \simeq\left\|D^{(n+q) / 2} g\right\|_{q} \simeq\left\|D^{n / 2} g\right\|_{0}
$$

for every $g \in H(B)$ and every $q \geq 0$.

THEOREM 4.1. For any $q \geq 0, \mathrm{HS}_{q}(B)=A_{q,(n+q) / 2}^{2}=\mathrm{HS}(B)=A_{0, n / 2}^{2}$ and the corresponding norms are equivalent.

Proof. Let $q \geq 0$ and $g \in H(B)$ with

$$
g(z)=\sum_{\alpha} b_{\alpha} z^{\alpha} \quad(z \in B) .
$$

Thus $K_{g}^{(q)}(f)=P_{q}(g \bar{f}), f \in A_{q}^{2}$, where $P_{q}$ is the orthogonal projector of $L_{q}^{2}$ onto $A_{q}^{2}$. Since $k_{q}(z, \zeta)=(1-\langle z, \zeta\rangle)^{-(n+q)}$ is the reproducing kernel of $A_{q}^{2}$ we find that $\left\{\phi_{\alpha}\right\}$, given by

$$
\phi_{\alpha}(z)=\sqrt{(n+q)_{|\alpha|} / \alpha !} z^{\alpha} \quad\left(\alpha \in \mathbf{Z}_{+}^{n}\right)
$$

is an orthonormal basis for $A_{q}^{2}$. Now, by a direct calculation (see also [5]),

$$
\begin{aligned}
\left\{K_{g}^{(q)}\left(\phi_{\alpha}\right)\right\} & =\left\{P_{q}\left(g \bar{\phi}_{\alpha}\right)\right\}(z)=\left\langle g \bar{\phi}_{\alpha}, k_{q}(\cdot, z)\right\rangle_{q} \\
= & \left(\frac{(n+q)_{|\alpha|}}{\alpha !}\right)^{1 / 2} \sum_{\gamma \geq 0}\left(\frac{(n+q)_{|\gamma|}}{\gamma !}\right)^{1 / 2} \frac{(a+\gamma) !}{(n+q)_{|\alpha+\gamma|}} b_{\alpha+\gamma} \phi_{\gamma}(z)
\end{aligned}
$$

and thus

$$
\left\langle K_{g}^{(q)}\left(\phi_{\alpha}\right), \phi_{\beta}\right\rangle_{q}=\left(\frac{(n+q)_{|\alpha|}(n+q)_{|\beta|}}{\alpha ! \beta !}\right)^{1 / 2} \frac{(\alpha+\beta) !}{(n+q)_{|\alpha+\beta|}} b_{\alpha+\beta} .
$$

It follows that the Hilbert-Schmidt norm is

$$
\begin{aligned}
\left\|K_{g}^{(q)}\right\|_{2}^{2} & =\sum_{\alpha, \beta \geq 0}\left|\left\langle K_{g}^{(q)}\left(\phi_{\alpha}\right), \phi_{\beta}\right\rangle_{q}\right|^{2} \\
& =\sum_{\alpha \geq 0} \frac{(n+q)_{|\alpha|}}{\alpha !} \sum_{\beta \geq 0} \frac{(n+q)_{|\beta|}}{\beta !}\left(\frac{(\alpha+\beta) !}{(n+q)_{|\alpha+\beta|}}\right)^{2}\left|b_{\alpha+\beta}\right|^{2} \\
& =\sum_{\alpha \geq 0} \frac{(n+q)_{|\alpha|}}{\alpha !} \sum_{\beta \geq \alpha} \frac{(n+q)_{|\beta-\alpha|}}{(\beta-\alpha) !}\left(\frac{\beta !}{(n+q)_{|\beta|}}\right)^{2}\left|b_{\beta}\right|^{2} \\
& =\sum_{\alpha \geq 0}\left(\frac{\alpha !}{(n+q)_{|\alpha|}}\right)^{2}\left|b_{\alpha}\right|^{2} \sum_{0 \leq \beta \leq \alpha} \frac{(n+q)_{|\beta|}}{\beta !} \cdot \frac{(n+q)_{|\alpha-\beta|}}{(\alpha-\beta) !}
\end{aligned}
$$


or

$$
\left\|K_{g}^{(q)}\right\|_{2}^{2}=\sum_{\alpha} \frac{\alpha !}{(n+q)_{|\alpha|}} \cdot \frac{(2 n+2 q)}{(n+q)_{|\alpha|}}\left|b_{\alpha}\right|^{2} .
$$

On the other hand, by (2.3)

$$
\|g\|_{2, q,(n+q) / 2}^{2}=\sum_{\alpha} \frac{\alpha !}{(n+q)_{|\alpha|}}(|\alpha|+1)^{n+q}\left|b_{\alpha}\right|^{2} .
$$

It follows from Stirling's formula that

$$
\left\|K_{g}^{(q)}\right\|_{2} \simeq\|g\|_{2, q,(n+q) / 2} \simeq\|g\|_{2,0, n / 2} \simeq\left\|K_{g}\right\|_{2},
$$

and the proof is complete.

We also note that formula (4.1) in the above proof shows that the space $\operatorname{HS}_{q}(B)$, with the natural inner product induced from the corresponding Hilbert-Schmidt norm, is a functional Hilbert space of functions on $H(B)$ with the reproducing kernel $h_{q}$, given by

$$
h_{q}(z, \zeta)=F(n+q, n+q ; 2 n+2 q:\langle z, \zeta\rangle) \quad(z, \zeta \in B),
$$

where

$$
F(a, b ; c: \lambda)=\sum_{m=0}^{\infty} \frac{(a)_{m}(b)_{m}}{m !(c)_{m}} \lambda^{m} \quad(\lambda \in \Delta)
$$

is the familiar hypergeometric function (see $[4,5])$. In particular,

$$
\begin{aligned}
F(n+q, n+q ; 2 n+2 q: \lambda) & \\
= & \frac{\Gamma(2 n+2 q)}{\{\Gamma(n+q)\}^{2}} \int_{0}^{1}(1-\lambda t)^{-(n+q)}\{t(1-t)\}^{n+q-1} d t,
\end{aligned}
$$

and thus, when $n=1$ the spaces $\operatorname{HS}(\Delta), A_{0,1 / 2}^{2}$ and $A_{1,1}^{2}(\Delta)$ are identical, as functional Hilbert spaces, with the reproducing kernel

$$
\begin{aligned}
F(1,1 ; 2: \lambda \bar{\mu}) & =G_{1,1}(\lambda \bar{\mu})=G_{2,2}(\lambda \bar{\mu}) \\
& =-\{\lambda \bar{\mu}\}^{-1} \log (1-\lambda \bar{\mu}), \quad \lambda, \mu \in \Delta .
\end{aligned}
$$

Moreover, one can show that the asymptotic behavior of

$$
F(n+q, n+q ; 2 n+2 q: \lambda)
$$

is similar to that of $G_{n+q, n+q}(\lambda)$ as described in Lemma 3.1 (i). In fact, for $\lambda \in \Delta$,

$$
\begin{aligned}
F(n+q, n+q ; & 2 n+2 q: \lambda) \\
= & -\Gamma(2 n+2 q)\left\{\Gamma^{2}(n+q) \lambda\right\}^{-1} \log (1-\lambda)+F_{q}(\lambda),
\end{aligned}
$$

where $F_{q}$ is in $\Lambda_{1}(\Delta)$ with $F_{q}(0)=1-\Gamma(2 n+2 q) /\{\Gamma(n+q)\}^{2}$.

We are now in a position to prove assertion (ii) of Theorem 2.8. 
THEOREM 4.2. Let $0<p \leq 2, q \geq 0$ and $s=(n+q) / p$. Then $A_{q, s}^{p} \subset$ $\mathrm{HS}(B)$ and the inclusion is continuous.

Proof. By Theorem 2.5 (iii) and Theorem 2.6, $A_{q, s}^{p} \subset A_{0, n / 2}^{2}$ and the inclusion is continuous. But by Theorem 4.1, $A_{0, n / 2}^{2}=\mathrm{HS}(B)$ and the norms are equivalent. This concludes the proof.

5. Sharp Refinement. In this section we consider the question of the sharpness of the refinement provided by Theorem 2.8. For this purpose, we define a holomorphic function $h_{s, t}, s, t \in \mathbf{R}$, on the unit disk $\Delta$ by

$$
h_{s, t}(\lambda)=(1-\lambda)^{-s}\left\{\frac{1}{\lambda} \log \frac{1}{1-\lambda}\right\}^{t}
$$

and we let

$$
h_{s, t}(\lambda)=\sum_{m=0}^{\infty} a_{m}(s, t) \lambda^{m} .
$$

Thus $a_{0}(s, t)=h_{s, t}(0)=1$. This function satisfies the identity

$$
D_{s+t} h_{s, t}=t h_{s+1, t-1}+s h_{s+1, t}
$$

and thus

$$
D_{t} h_{0, t}=t h_{1, t-1} \text {. }
$$

(Recall that $D_{t}=t+R$ and that $D=d_{1}$.)

LEMMA 5.1. For $m=1,2, \ldots$, we have

$$
D_{t}^{m} h_{0, t}=c_{0 m} h_{m, t-m}+\sum_{i=1}^{m-1} \sum_{j=1}^{i} c_{i j} h_{i+1, t-j}
$$

where the $c_{i j}$ are functions of $t$ with $c_{0 m}=t(t-1) \cdots(t-m+1)$ and $c_{11}=(-1)^{m} t$.

Proof. For $m=1$ this formula reduces to (5.2), while for $m \geq 2$ the formula follows from the identity (5.1) and induction on $\mathrm{m}$.

For $0<p<\infty$ and $f \in H(\Delta)$, we let

$$
M_{p}(f: r)=\left\{\frac{1}{2 \pi}\left|f\left(r e^{i \theta}\right)\right|^{p} d \theta\right\}^{1 / p} \quad(0 \leq r<1) .
$$


Since $M_{p}(f: \cdot)$ is a non-negative increasing function on $[0,1)$, we deduce that for any $q>0$

$$
\int_{0}^{1} M_{p}^{p}(f: r) d\left(1-r^{2}\right)^{q} \simeq \int_{1 / 2}^{1} M_{p}^{p}(f: r) d\left(1-r^{2}\right)^{q} .
$$

This formula is also correct when $q=0$, in which case it is interpreted as

$$
\sup \left\{M_{p}^{p}(f: r): r \in[0,1)\right\}=\sup \left\{M_{p}^{p}(f: r): r \in[1 / 2,1)\right\} .
$$

The proof of the estimates appearing in the next lemma can be found in Littlewood [11, pp. 93-96].

Lemma. 5.2. Let $s \geq 0$ and $t \in \mathbf{R}$ with $t>0$ if $s=0$. Then $h_{s, t} \notin$ $H^{\infty}(\Delta)$ and

$$
a_{m}(s, t) \simeq \begin{cases}(m+1)^{s-1}\{\log (m+1)\}^{t}, & s>0, t \in \mathbf{R} \\ (m+1)^{-1}\{\log (m+1)\}^{t-1}, & s=0, t>0\end{cases}
$$

for $m=1,2, \ldots$ Moreover, for $0<p<\infty$ and $r \in[1 / 2,1)$,

$$
M_{p}\left(h_{s, t}: r\right) \simeq \begin{cases}1, & p s<1 \text { or } p s=1, p t<-1 \\ \{-\log (1-r)\}^{t+1 / p}, & p s=1, p t>-1 \\ (1-r)^{-s+1 / p}\{-\log (1-r)\}^{t}, & p s>1 .\end{cases}
$$

We now prove the following theorem which establishes the sharpness of the refinement given in Theorem 2.8. Special cases of this result can be found also in Graham [8] and Beatrous [2].

THEOREM 5.3. Let $0<p<\infty, q \geq 0$ and $s=(n+q) / p$.

(i) If $p>2$ then $A_{q, s}^{p}$ contains unbounded functions which are also not in $\operatorname{HS}(B)$;

(ii) If $p>1$ then $A_{q, s}^{p}$ contains unbounded functions which are in $\operatorname{HS}(B)$.

Proof. To prove (i) we choose $p_{1}$ with $2<p_{1}<p$, and define $m=\left[n / p_{1}\right]+1$, where $[x]$ denotes the integer-value of $x$. It follows that $m>1$ is an integer with $m>n / p_{1}$. We now let $q_{1}=m p_{1}-n$, and thus $m=\left(n+q_{1}\right) / p_{1}$ with $q_{1}>0$. It follows from Theorem 2.6 that $A_{q_{1}, m}^{p_{1}} \subset$ $A_{q, s}^{p}$. We now let $f=h_{0,1 / 2} \circ u_{1}$, where $u_{1}$ is the orthogonal projection of $\mathbf{C}^{n}$ on the first coordinate. Since $h_{0,1 / 2}(\lambda)=\left\{-\lambda^{-1} \log (1-\lambda)\right\}^{1 / 2}$ it is clear that $f \notin H^{\infty}$. Similarly, since by Lemma $5.2 h_{0,1 / 2} \notin A_{0,1 / 2}^{2}(\Delta)$ we find that $f=h_{0,1 / 2} \circ u_{1} \notin A_{0, n / 2}^{2}$ and hence, by Theorem 4.1, also $f \notin$ $\operatorname{HS}(B)$. Thus, to prove (i), it suffices to show that $f \in A_{q_{1}, m}^{p_{1}}$, i.e. that 
$\left\|D^{m} f\right\|_{p_{1}, q_{1}}<\infty$. But (see [3]) $\left\|D^{m} f\right\|_{p_{1}, q_{1}} \simeq\left\|D_{1 / 2}^{m} f\right\|_{p_{1}, q_{1}}$, and hence it is sufficient to show that $\left\|D_{1 / 2}^{m} f\right\|_{p_{1}, q_{1}}<\infty$. Now, by Fubini's theorem (see also [12, pp. 127-128]).

$$
\left\|D_{1 / 2}^{m} f\right\|_{p_{1}, q_{1}}=\left\|D_{1 / 2}^{m} h_{0,1 / 2} \circ u_{1}\right\|_{p_{1}, q_{1}}=\left\|D_{1 / 2}^{m} h_{0,1 / 2}\right\|_{p_{1}, q_{1}+n-1} .
$$

while by Lemma 5.1 and the triangle-inequality

$$
\begin{aligned}
& \left\|D_{1 / 2}^{m} h_{0,1 / 2}\right\|_{p_{1}, q_{1}+n-1}=\left\|D_{1 / 2}^{m} h_{0,1 / 2}\right\|_{p_{1}, m p_{1}-1} \\
& \quad \leq\left|c_{0 m}\right|\left\|h_{m, 1 / 2-m}\right\|_{p_{1}, m p_{1}-1}+\sum_{i=1}^{m-1} \sum_{j=1}^{i}\left|c_{i j}\right|\left\|h_{i+1,1 / 2-j}\right\|_{p_{1}, m p_{1}-1},
\end{aligned}
$$

where $c_{0 m}=(-1)^{m}(-1 / 2)_{m}=\left(\begin{array}{c}1 / 2 \\ m\end{array}\right)$ and $c_{11}=(-1)^{m} / 2$. On the other hand by formula (5.3) and Lemma 5.2 we have, for every $i$ and $j$ with $1 \leq i \leq m$ and $j \geq 1$,

$$
\begin{aligned}
\left\|h_{i, 1 / 2-j}\right\|_{p_{1}, m p_{1}-1}^{p_{1}} & =-\int_{0}^{1} M_{p_{1}}^{p_{1}}\left(h_{i, 1 / 2-j}: r\right) d\left(1-r^{2}\right)^{m p_{1}-1} \\
& \simeq-\int_{1 / 2}^{1} M_{p_{1}}^{p_{1}}\left(h_{i, 1 / 2-j}: r\right) d\left(1-r^{2}\right)^{m p_{1}-1} \\
& \simeq \int_{1 / 2}^{1}\{-\log (1-r)\}^{p_{1}(1 / 2-j)}(1-r)^{p_{1}(m-i)-1} d r \\
& \leq 2^{p_{1}(i-m)} \int_{1 / 2}^{1}\{-\log (1-r)\}^{p_{1}(1 / 2-j)}(1-r)^{-1} d r \\
& =2^{p_{1}(i-m)}\left\{p_{1}(j-1 / 2)-1\right\}^{-1}(\log 2)^{p_{1}(1 / 2-j)+1}<\infty,
\end{aligned}
$$

since $p_{1}>2$. Thus, it follows that $\left\|D_{1 / 2}^{m} f\right\|_{p_{1}, q_{1}}<\infty$, and (i) is proved.

To prove (ii), we let $f=f_{s} \circ u_{1}$ where, for $s>0$,

$$
f_{s}(\lambda)=\frac{1}{\Gamma(s)} \int_{0}^{1} h_{s,-1}(\lambda u)\{-\log u\}^{s-1} d u \quad(\lambda \in \Delta) .
$$

It follows easily that $h_{s,-1}=D^{s} f_{s}$ or $f_{s}=D^{-s} h_{s,-1}$, and, in particular,

$$
f_{s}(\lambda)=\sum_{m=0}^{\infty}(m+1)^{-s} a_{m}(s,-1) \lambda^{m} \quad(\lambda \in \Delta) .
$$

Now, by Lemma 5.2,

$$
\begin{aligned}
\lim _{r \rightarrow 1^{-}} f(r, 0, \ldots, 0) & =\lim _{r \rightarrow 1^{-}} f_{s}(r)=\sum_{m=0}^{\infty}(m+1)^{-s} a_{m}(s,-1) \\
& \simeq 1+\sum_{m=2}^{\infty} \frac{1}{m \log m}=\infty
\end{aligned}
$$


and thus $f \notin H^{\infty}$. Moreover, again by Fubini's theorem, $\left\|D^{n / 2} f\right\|_{2,0}=$ $\left\|D^{n / 2} f_{s}\right\|_{2, n-1}$. But, by (5.4), (2.3), Lemma 5.2 and Stirling's formula,

$$
\begin{aligned}
\left\|D^{n / 2} f_{s}\right\|_{2, n-1}^{2} & =\sum_{m=0}^{\infty} \frac{m !}{(n)_{m}}(m+1)^{n-2 s}\left|a_{m}(s,-1)\right|^{2} \\
& \simeq 1+\sum_{m=1}^{\infty} \frac{m !}{(n)_{m}}(m+1)^{n-2}\{\log (m+1)\}^{-2} \\
& \simeq 1+\sum_{m=2}^{\infty} \frac{1}{m(\log m)^{2}}<\infty .
\end{aligned}
$$

It follows that $f \in A_{0, n / 2}^{2}$, and, since, by Theorem 4.1, $A_{0, n / 2}^{2}=\operatorname{HS}(B)$ with equivalent norms, we deduce that $f \in \operatorname{HS}(B)$ and $f \notin H^{\infty}$. To complete the proof, we must show, therefore, that $f \in A_{q, s}^{p}$. Now, once again, by Fubini's theorem,

$$
\left\|D^{s} f\right\|_{p, q}=\left\|h_{s,-1} \circ u_{1}\right\|_{p, q}=\left\|h_{s,-1}\right\|_{p, q+n-1}=\left\|h_{s,-1}\right\|_{p, p s-1} .
$$

Here, by assumption, $p s-1 \geq 0$ and $p s-1=0$ if and only if $n=1$ and $q=0$. Thus, by formula (5.3) (including its modification for $q=0$ ) with $p s-1$ in place of $q$, and by Lemma 5.2, we obtain

$$
\begin{aligned}
\left\|h_{s,-1}\right\|_{p, p s-1}^{p} & =-\int_{0}^{1} M_{p}^{p}\left(h_{s,-1}: r\right) d\left(1-r^{2}\right)^{p s-1} \\
& \simeq-\int_{1 / 2}^{1} M_{p}^{p}\left(h_{s,-1}: r\right) d\left(1-r^{2}\right)^{p s-1} \\
& \simeq-\int_{1 / 2}^{1}\{-\log (1-r)\}^{-p}(1-r)^{-1} d r \\
& =(p-1)^{-1}(\log 2)^{1-p}<\infty,
\end{aligned}
$$

since $p>1$. This is also correct when $p s=1$, for in this case, by formula (5.3) and Lemma 5.2, $\left\|h_{s,-1}\right\|_{p, 0}=\sup \left\{M_{p}\left(h_{1 / p,-1}: r\right): r \in[0,1)\right\}=$ $\sup \left\{M_{p}\left(h_{1 / p,-1}: r\right): r \in[1 / 2,1)\right\} \simeq 1<\infty$. The proof is now complete.

\section{REFERENCES}

[1] F. Beatrous, Estimates for derivatives of holomorphic functions in pseudoconvex domains, Math. Z., 191 (1986), 91-116.

[2] , Boundary continuity of holomorphic functions in the ball, Proc. Amer. Math. Soc., 97 (1986), 23-29.

[3] F. Beatrous and J. Burbea, Sobolev spaces of holomorphic functions in the ball, Pitman Research Notes in Math., Pitman, London, to appear.

[4] J. Burbea, Norm inequalities of exponential type of holomorphic functions, Kōdai Math. J., 5 (1982), 339-354. 
[5] - Inequalities for holomorphic functions of several complex variables, Trans. Amer. Math. Soc., 276 (1983), 247-266.

[6] R. R. Coifman, R. Rochberg and G. Weiss, Factorization theorems for Hardy spaces in several variables, Ann. Math., 103 (1976), 611-635.

[7] P. L. Duren, Theory of $H^{p}$ Spaces, Academic Press, New York, 1970.

[8] I. Graham, An $H^{p}$ theorem for the radial derivatives of holomorphic functions on the unit ball in $\mathbf{C}^{n}$, preprint.

[9] - The radial derivative, fractional integrals, and the comperative growth of holomorphic functions on the unit ball in $\mathbf{C}^{n}$, Recent Developments in Several Complex Variables, Annals Math. Studies, 100 (1981), 171-178.

[10] S. G. Krantz, Analysis on the Heisenberg group and estimates for functions in Hardy classes of several complex variables, Math. Ann., 244 (1979), 243-262.

[11] J. E. Littlewood, Lectures on the Theory of Functions, Oxford Univ. Press, London, 1944.

[12] W. Rudin, Function Theory in the Unit Ball of $\mathbf{C}^{n}$, Springer-Verlag, New York, 1980.

[13] D. Sarason, Functions of vanishing mean oscillation, Trans. Amer. Math. Soc., 207 (1975), 391-405.

[14] D. A. Stegenga, Bounded Toeplitz operators on $H^{1}$ and applications of the duality between $H^{1}$ and the functions of bounded mean oscillation, Amer. J. Math., 98 (1976), 573-589.

Received November 22, 1985.

UNIVERSity OF PitTSBURgh

PitTSBURgh, PA 15260 



\section{PACIFIC JOURNAL OF MATHEMATICS EDITORS}

\author{
V. S. VARADARAJAN \\ (Managing Editor) \\ University of California \\ Los Angeles, CA 90024 \\ Herbert Clemens \\ University of Utah \\ Salt Lake City, UT 84112 \\ R. FINN \\ Stanford University \\ Stanford, CA 94305
}

\author{
HERMANN FLASCHKA \\ University of Arizona \\ Tucson, AZ 85721 \\ RAMESh A. GANGOLli \\ University of Washington \\ Seattle, WA 98195 \\ VAUGHAN F. R. JONES \\ University of California \\ Berkeley, CA 94720 \\ ROBION KIRBY \\ University of California \\ Berkeley, CA 94720 \\ C. C. MOORE \\ University of California \\ Berkeley, CA 94720 \\ H. SAMELSON \\ Stanford University \\ Stanford, CA 94305 \\ HAROLD STARK \\ University of California, San Diego \\ La Jolla, CA 92093
}

\section{ASSOCIATE EDITORS}
R. ARENS
E. F. BECKENBACH
B. H. NEUMANN
F. WOLF
K. YOSHIDA (1906-1982)

\section{SUPPORTING INSTITUTIONS}
UNIVERSITY OF ARIZONA
UNIVERSITY OF BRITISH COLUMBIA
CALIFORNIA INSTITUTE OF TECHNOLOGY
UNIVERSITY OF CALIFORNIA
MONTANA STATE UNIVERSITY
UNIVERSITY OF NEVADA, RENO
NEW MEXICO STATE UNIVERSITY
OREGON STATE UNIVERSITY
UNIVERSITY OF OREGON UNIVERSITY OF SOUTHERN CALIFORNIA STANFORD UNIVERSITY UNIVERSITY OF HAWAII UNIVERSITY OF TOKYO UNIVERSITY OF UTAH WASHINGTON STATE UNIVERSITY UNIVERSITY OF WASHINGTON 


\section{Pacific Journal of Mathematics \\ Vol. 127, No. $1 \quad$ January, 1987}

Jacob Burbea, Boundary behavior of holomorphic functions in the ball ..... 1

Jan Dijkstra, Strong negligibility of $\sigma$-compacta does not characterize

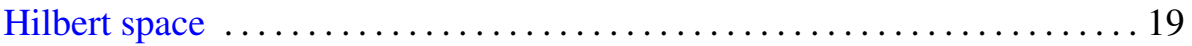

Ruy Exel, Rotation numbers for automorphisms of $C^{*}$ algebras $\ldots \ldots \ldots 31$

Howard Jacobowitz, The canonical bundle and realizable CR

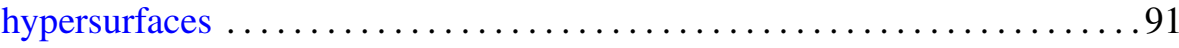

James T. Joichi and Dennis Warren Stanton, Bijective proofs of basic

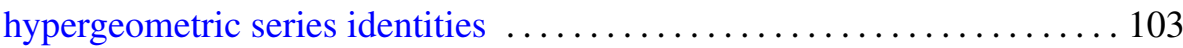

Gareth J. Knowles, Quotients of nest algebras with trivial commutator .... 121

Murray Angus Marshall, Exponentials and logarithms on Witt rings ..... 127

Courtney Hughes Moen, The dual pair $(U(3), U(1))$ over a $p$-adic field . ...141

William Ortmeyer, Surgery on a class of pretzel knots $\ldots \ldots \ldots \ldots \ldots \ldots 155$

John Gerard Ryan, Extensions of representations of Lie algebras . . . . . 173

Ivan Charles Sterling, A generalization of a theorem of Delaunay to

rotational $W$-hypersurfaces of $\sigma_{l}$-type in $H^{n+1}$ and $S^{n+1} \ldots \ldots \ldots 187$

Vesko M. Valov, Another characterization of AE(0)-spaces $\ldots \ldots \ldots \ldots \ldots 9$ 